\title{
CAPTURE: a multinational, cross-sectional study of cardiovascular disease prevalence in adults with type 2 diabetes across 13 countries
}

Ofri Mosenzon ${ }^{1 *} \mathbb{C}$, Abdullah Alguwaihes ${ }^{2}$, Jose Luis Arenas Leon ${ }^{3}$, Fahri Bayram4 ${ }^{4}$ Patrice Darmon ${ }^{5}$, Timothy M. E. Davis ${ }^{6}$, Guillermo Dieuzeide ${ }^{7}$, Kirsten T. Eriksen ${ }^{8}$, Tianpei Hong ${ }^{9}$, Margit S. Kaltoft ${ }^{8}$, Csaba Lengyel ${ }^{10}$, Nicolai A. Rhee ${ }^{11}$, Giuseppina T. Russo ${ }^{12}$, Shinichiro Shirabe ${ }^{13}$, Katerina Urbancova ${ }^{14}$ and Sergio Vencio ${ }^{15}$ on behalf of the CAPTURE Study Investigators

\begin{abstract}
Background: There is a paucity of global data on cardiovascular disease (CVD) prevalence in people with type 2 diabetes (T2D). The primary objective of the CAPTURE study was to estimate the prevalence of established CVD and its management in adults with T2D across 13 countries from five continents. Additional objectives were to further characterize the study sample regarding demographics, clinical parameters and medication usage, with particular reference to blood glucose-lowering agents (GLAs: glucagon-like peptide-1 receptor agonists and sodium-glucose co-transporter-2 inhibitors) with demonstrated cardiovascular benefit in randomized intervention trials.

Methods: Data were collected from adults with T2D managed in primary or specialist care in Australia, China, Japan, Czech Republic, France, Hungary, Italy, Argentina, Brazil, Mexico, Israel, Kingdom of Saudi Arabia, and Turkey in 2019, using standardized methodology. CVD prevalence, weighted by diabetes prevalence in each country, was estimated for the overall CAPTURE sample and participating countries. Country-specific odds ratios for CVD prevalence were further adjusted for relevant demographic and clinical parameters.

Results: The overall CAPTURE sample included 9823 adults with T2D ( $n=4502$ from primary care; $n=5321$ from specialist care). The overall CAPTURE sample had median (interquartile range) diabetes duration 10.7 years (5.617.9 years) and glycated hemoglobin $7.3 \%(6.6-8.4 \%)[56 \mathrm{mmol} / \mathrm{mol}(49-68 \mathrm{mmol} / \mathrm{mol})]$. Overall weighted CVD and atherosclerotic CVD prevalence estimates were $34.8 \%$ (95\% confidence interval [Cl] $32.7-36.8)$ and $31.8 \%(95 \% \mathrm{Cl}$ 29.7-33.8\%), respectively. Age, gender, and clinical parameters accounted for some of the between-country variation in CVD prevalence. GLAs with demonstrated cardiovascular benefit were used by $21.9 \%$ of participants, which was similar in participants with and without CVD: $21.5 \%$ and $22.2 \%$, respectively.
\end{abstract}

\footnotetext{
*Correspondence: ofrim@hadassah.org.il

${ }^{1}$ Diabetes Unit, Department of Endocrinology and Metabolism, Hadassah Medical Center, Faculty of Medicine, Hebrew University of Jerusalem, Hadassah Hebrew University Hospital, PO Box 12000, 91120 Jerusalem, Israel

Full list of author information is available at the end of the article
} permits use, sharing, adaptation, distribution and reproduction in any medium or format, as long as you give appropriate credit to the original author(s) and the source, provide a link to the Creative Commons licence, and indicate if changes were made. The images or other third party material in this article are included in the article's Creative Commons licence, unless indicated otherwise in a credit line to the material. If material is not included in the article's Creative Commons licence and your intended use is not permitted by statutory regulation or exceeds the permitted use, you will need to obtain permission directly from the copyright holder. To view a copy of this licence, visit http://creativecommons.org/licenses/by/4.0/. The Creative Commons Public Domain Dedication waiver (http://creativeco mmons.org/publicdomain/zero/1.0/) applies to the data made available in this article, unless otherwise stated in a credit line to the data. 
Conclusions: In 2019, approximately one in three adults with T2D in CAPTURE had diagnosed CVD. The low use of GLAs with demonstrated cardiovascular benefit even in participants with established CVD suggested that most were not managed according to contemporary diabetes and cardiology guidelines.

Study registration NCT03786406 (registered on December 20, 2018), NCT03811288 (registered on January 18, 2019).

Keywords: Non-interventional study, Type 2 diabetes, Cardiovascular disease, Atherosclerotic cardiovascular disease, Prevalence, Glucagon-like peptide-1 receptor agonists, Sodium-glucose co-transporter-2 inhibitors

\section{Introduction}

Cardiovascular disease (CVD) remains the largest cause of diabetes-related morbidity and mortality [1,2]. Systematic reviews indicate there is an approximately twofold higher risk of CVD in people with versus without diabetes after adjustment for conventional risk factors [3, 4]. Given the large clinical burden associated with CVD complicating type 2 diabetes (T2D), international diabetes and cardiology position statements and guidelines have been updated to encompass the combined management of T2D and CVD [5-7]. These updates have been informed by cardiovascular (CV) outcome trials demonstrating superiority in $\mathrm{CV}$ outcomes for some blood glucose-lowering agents (GLAs) versus placebo in people with T2D and established CVD or at high CVD risk [6, 8]. The glucagon-like peptide-1 receptor agonists (GLP-1 RAs) and sodium-glucose co-transporter- 2 inhibitors (SGLT2is) with demonstrated CV benefit are now recommended as first [5] or second-line [6, 7] GLAs in this context.

The impact of the updated guidelines on real-world clinical practice should be of interest to clinicians and policy makers, and its evaluation requires robust contemporary estimates of CVD prevalence and management in people with T2D that can be monitored over time. However, the current understanding of the global prevalence of, and between-country variation in, diabetes-related complications, including CVD, is limited [9]. Available CVD prevalence data in people with T2D are mostly from regional or national studies conducted in the US [10] and some European countries [11-15]. Direct comparisons between countries are complicated by differences in study methodology, including the population sampled and definition of CVD. Estimates of the CVD burden in some countries have been based on studies that sampled selected patient populations (such as from specialist care) or that utilized model-based estimates rather than real-world data [16]. A review paper estimated CVD to affect $32.2 \%$ of individuals with T2D worldwide, based on a systematic review of scientific literature published between 2007 and 2017 [16] that would not reflect recent changes in epidemiology and management of T2D and is now considered outdated.
The primary objective of the present study was to estimate the contemporary prevalence of established CVD in adults with T2D across a range of countries and several continents using standardized methodology. Secondary objectives were to estimate the prevalence of high CVD risk in adults with T2D without established CVD (full results to be published elsewhere) and to further characterize the study sample regarding demographics, clinical parameters, CV medication and GLA usage, with particular reference to GLAs (GLP-1 RAs and SGLT2is) with demonstrated CV benefit.

\section{Methods}

Study design

CAPTURE was a non-interventional, cross-sectional study conducted at 214 centers across 13 countries (NCT03786406 [Europe] and NCT03811288 [non-Europe]). The countries were from Australasia (Australia), East Asia (China and Japan), Europe (Czech Republic, France, Hungary, and Italy), Latin America (Argentina, Brazil, and Mexico), and the Middle East (Israel, Kingdom of Saudi Arabia and Turkey). The study protocols were approved by the appropriate independent ethics committees and relevant institutional review boards. The study was conducted in accordance with the Declaration of Helsinki [17], International Society for Pharmacoepidemiology Good Pharmacoepidemiology Practices [18], and local regulations. All participants provided written informed consent prior to participation.

Local medical affairs personnel from participating countries, who were employed by the sponsor, provided information on the management of people with T2D, including types of physicians managing T2D in routine clinical practice (primary care practitioners, diabetologists, endocrinologists, cardiologists, and other specialists) and types of practices (primary care and specialist settings, including different types of hospitals) to a contract research organization for consideration of site selection. Final participating sites were chosen by the contract research organization and approved by the sponsor on the basis that they were as representative as possible for each country. Factors considered were geographical spread and the division of patients being treated at 
private and public centers, as well as the degree of specialization at each site. The ratio of participants from primary to specialist care sites in each country was chosen to approximate the assumed distribution of adults with T2D managed in these settings according to available local data. No primary care sites were included in China, Hungary, or Italy as people with T2D in these countries are primarily, although not exclusively, managed in specialist care. In Italy, local health system organization rules mean that new prescriptions for GLAs cannot be provided in primary care. In China, patients with T2D consult hospital doctors as their primary contact. In Hungary, patients with T2D are mainly managed in a specialist care setting with limited influence from primary care on decisions regarding GLAs with CV benefit.

At each participating site, consecutive, eligible adults aged $\geq 18$ years ( $\geq 20$ years in Japan) at the time of informed consent who had been diagnosed with $\mathrm{T} 2 \mathrm{D} \geq 180$ days prior to providing informed consent and who were attending the site as part of their routine visit to their treating physician were invited to participate by the treating physician during a 90-day time window. Exclusion criteria were a diagnosis of type 1 diabetes, known congenital heart disease or malformation, previous participation in this study (provided informed consent for inclusion at a prior visit during the data collection period), and mental incapacity or language barriers that precluded an adequate understanding of, or cooperation with, study requirements.

The invitation to participate, provision of informed consent, and data collection took place during a single, routine health visit at each site. In all countries, the treating physician or a trained delegate collected data using a standardized electronic case report form, and data were transferred to a central database via a web-based data capture system. Relevant data were collected from participants' medical records. The physician verbally asked participants for any information that was missing from the medical record. The study protocols did not mandate screening for, or adjudication of, the presence of complications .

\section{Definitions of variables studied}

Established CVD was defined as a diagnosis of any of the following conditions in participants' medical records: cerebrovascular disease, coronary heart disease (CHD), heart failure, cardiac arrhythmia or conduction abnormalities, aortic disease, peripheral artery disease (PAD), or carotid artery disease (see Additional file 1: Table S1 for the full list and definitions). Similarly, CVD was categorized as atherosclerotic CVD (ASCVD) when there was a diagnosis of any of the following $\mathrm{CV}$ conditions: cerebrovascular disease, CHD, PAD, or carotid artery disease [7]. For analysis purposes, participants were stratified into two groups based on the presence (CVD group) or absence (non-CVD group) of established CVD.

Available demographic, anthropometric, and clinical parameters were collected, in addition to selected medical history, GLAs, and CV medications (listed in Additional file 1: Methods S1). Only current medications or those discontinued within the previous 3 months were recorded. During analysis, GLAs were further grouped according to demonstrated CV benefit status in line with the 2020 American Diabetes Association guidelines [19] (and by March 2020, all had a CV indication in their US Food and Drug Administration label [20-25]). GLAs with demonstrated $\mathrm{CV}$ benefit included three GLP-1 RAs (dulaglutide, liraglutide, and semaglutide) and three SGLT2is (canagliflozin, dapagliflozin, and empagliflozin).

\section{Statistical analysis \\ Pre-specified analyses}

The prevalence (95\% confidence interval $[\mathrm{CI}]$ ) of CVD, ASCVD, CVD subtypes, and diagnoses were estimated for all countries together (overall) and for each country individually. Overall prevalence estimates were calculated as weighted estimates to account for the size of the diabetes population in each country [26], as this was not accounted for in the sampling. Both the overall and country-level prevalence estimates were calculated as weighted estimates to account for any differences between the actual and planned sampling distribution of participants by healthcare setting (primary care:specialist care).

The study sample was characterized by demographics, clinical parameters, CV medication, and GLA usage, with particular reference to GLAs with demonstrated CV benefit, presented for the overall study sample and separately for the CVD group and non-CVD group; data were not weighted.

\section{Post hoc analyses}

The number of affected vascular areas among those with CVD was calculated and stratified by gender. Three vascular areas were analyzed, defined as coronary, cerebrovascular and peripheral.

To explore whether differences in estimated CVD prevalence between countries could be partially or fully explained by differences in demographic and clinical characteristics between the country samples, logistic regression models were used to calculate prevalence odds ratios (PORs) for CVD in each country using the overall CAPTURE study sample as the reference. The models were as follows: (1) crude; (2) adjusted for 
age and gender; and (3) additionally adjusted for statistically significant clinical parameters identified via backwards selection (age, gender, diabetes duration, body mass index $[\mathrm{BMI}]$, glycated hemoglobin $\left[\mathrm{HbA}_{1 \mathrm{c}}\right]$, low-density lipoprotein cholesterol, high-density lipoprotein cholesterol, smoking status, hypertension, nephropathy, neuropathy, and retinopathy). Parameters with a high proportion of missing data (albuminuria, estimated glomerular filtration rate [eGFR] and physical activity) were not considered (Additional file 1: Table S2). Any missing data for the included parameters were imputed for each country separately using fully conditional specification. Sensitivity analyses were conducted (1) without imputation of missing data for participants with complete covariate information and (2) including eGFR as a clinical parameter (both with and without imputation of missing data). Further details on the logistic regression analyses are available in Additional file 1: Methods S2.

Due to the large T2D population compared to the other study countries, China would have a large influence on the overall weighted CVD prevalence estimates. As such, a sensitivity analysis was conducted where the prevalence calculations were repeated for all countries excluding China. These estimates were also calculated as weighted estimates to account for the size of the diabetes population of the 12 remaining countries [26].

In order to place the CAPTURE data in the context of two recent $\mathrm{CV}$ outcome trials, exploratory analyses assessed the number of participants with high CVD risk in the non-CVD group who were using a GLA with demonstrated CV benefit. Participants with high CVD risk were identified in the non-CVD group as satisfying relevant criteria from the REWIND [27] or DECLARETIMI 58 [28] trials based on available CAPTURE data. To align as closely as possible with REWIND criteria, participants in the non-CVD group were categorized as being at high CVD risk if they were $\geq 60$ years of age; were a current (any self-reported current tobacco use) or previous smoker (any self-reported historical tobacco use); and were on anti-hypertensive medication or had elevated systolic blood pressure $(\geq 140 \mathrm{mmHg}$ ) or had elevated diastolic blood pressure ( $\geq 95 \mathrm{mmHg}$ ) [27]. To align as closely as possible with DECLARE-TIMI 58 criteria, participants in the nonCVD group were categorized as being at high CVD risk if they were $\geq 55$ years of age if male or $\geq 60$ years of age if female; and were either on anti-hypertensive medication or had both elevated systolic blood pressure $(>140 \mathrm{mmHg}$ ) and elevated diastolic blood pressure (>90 mmHg) [28].
All statistical analyses were carried out using SAS, Version 9.4 (SAS Institute, Cary, NC, USA).

\section{Results \\ Study sample}

The overall study sample included 9823 adults with T2D ( $n=4502$ from primary care; $n=5321$ from specialist care) (Additional file 1: Fig. S1 for study flow) who participated between December 01, 2018 and September 30, 2019. The median (interquartile range [IQR]) number of participants per site was 40 (25-57). The number and geographical distribution of study participants are presented in Additional file 1: Fig. S2. The median (IQR) age of the overall study sample was 64.0 years $(56.0-71.0$ years) and $45.5 \%$ of participants were female (Table 1 ; additional data in Additional file 1: Table S3). Most (80.4\%) participants had $\mathrm{a} B \mathrm{BMI} \geq 25 \mathrm{~kg} / \mathrm{m}^{2}$, and $70.1 \%$ had diagnosed hypertension. The median (IQR) $\mathrm{HbA}_{1 \mathrm{c}}$ was $7.30 \%$ (6.60-8.40\%) [56 mmol/mol (49-68 mmol/mol)] and diabetes duration was 10.7 years $(5.6-17.9$ years).

\section{CVD prevalence}

Among the 9823 study participants, over one third ( $\mathrm{n}=3582$; 36.5\%) had established CVD, with a weighted CVD prevalence estimated at 34.8\% (95\% CI 32.7-36.8\%) across the 13 countries. CVD prevalence was lowest in the Kingdom of Saudi Arabia (18.0\%) and highest in Israel (56.5\%) (Fig. 1). Most (85.8\%; $\mathrm{n}=3074$ ) cases of CVD were atherosclerotic, with the weighted ASCVD prevalence estimated at $31.8 \%$ (95\% CI 29.7-33.8\%) across the 13 countries. The weighted prevalence of CVD subtypes and diagnoses are presented in Fig. 2 and Additional file 1: Table S4. The most prevalent weighted CVD subtypes were CHD (17.7\%), carotid artery disease (8.4\%), and cerebrovascular disease (7.2\%). The overall weighted prevalence of heart failure was $2.4 \%$ (95\% CI $2.1-2.7 \%$ ), with a wide range from $0.2 \%$ in China to $14.5 \%$ in Israel (Additional file 1: Fig. S3). Overall, the weighted prevalence of CVD was similar in participants from primary versus specialist care (35.6\% vs $34.6 \%$ ) (not statistically analyzed).

In post hoc analyses that excluded China from the overall prevalence estimates, the weighted CVD prevalence was $36.6 \%$ (95\% CI 35.4-37.7\%) and heart failure prevalence was $6.9 \%$ (95\% CI 6.3-7.6\%) across the 12 remaining countries.

\section{Characteristics of the study sample by CVD status}

In comparison with the non-CVD group, the CVD group was older (median age 68.0 years vs 62.0 years), had a higher proportion of males (61.3\% vs $50.7 \%)$, and a longer duration of diabetes (median duration 13.0 years 
Table 1 Demographic and clinical characteristics of the CAPTURE study sample overall and stratified by CVD status

\begin{tabular}{|c|c|c|c|c|c|c|}
\hline \multirow[t]{3}{*}{ Characteristic } & \multirow{2}{*}{\multicolumn{2}{|c|}{$\begin{array}{l}\text { Overall } \\
\mathrm{N}=9823\end{array}$}} & \multicolumn{4}{|c|}{ By CVD status } \\
\hline & & & \multicolumn{2}{|c|}{$\begin{array}{l}\text { CVD } \\
n=3582\end{array}$} & \multicolumn{2}{|c|}{$\begin{array}{l}\text { Non-CVD } \\
n=6241\end{array}$} \\
\hline & $\mathbf{n}$ & Data & $\mathbf{n}$ & Data & $\mathbf{n}$ & Data \\
\hline Female, n (\%) & 9823 & $4465(45.5)$ & 3582 & $1388(38.7)$ & 6241 & $3077(49.3)$ \\
\hline Age, years, median [IQR] & 9823 & $64.0[56.0-71.0]$ & 3582 & $68.0[61.0-75.0]$ & 6241 & $62.0[54.0-69.0]$ \\
\hline Race, n (\%) & 9822 & & 3581 & & 6241 & \\
\hline White & & $6487(66.0)$ & & $2558(71.4)$ & & $3929(63.0)$ \\
\hline Asian & & $2133(21.7)$ & & $718(20.1)$ & & $1415(22.7)$ \\
\hline Black or African American & & $158(1.6)$ & & $66(1.8)$ & & $92(1.5)$ \\
\hline Other & & $1044(10.6)$ & & $239(6.7)$ & & $805(12.9)$ \\
\hline Diabetes duration, years, median [IQR] & 9811 & $10.7[5.6-17.9]$ & 3577 & $13.0[7.2-20.0]$ & 6234 & $9.8[4.8-15.9]$ \\
\hline $\mathrm{HbA}_{1 \mathrm{c}} \%$, median [IQR] & 9104 & $7.30[6.60-8.40]$ & 3289 & $7.40[6.60-8.50]$ & 5815 & $7.30[6.50-8.30]$ \\
\hline $\mathrm{HbA}_{1,}, \mathrm{mmol} / \mathrm{mol}$, median [IQR] & 9104 & $56[49-68]$ & 3289 & $57[49-69]$ & 5815 & $56[48-67]$ \\
\hline FPG, mmol/L, median [IQR] & 8204 & 7.60 [6.30-9.38] & 2924 & $7.60[6.21-9.43]$ & 5280 & $7.60[6.33-9.32]$ \\
\hline Body weight, kg, median [IQR] & 9742 & $79.3[68.7-92.0]$ & 3550 & $79.8[69.0-92.0]$ & 6192 & $79.0[68.3-92.0]$ \\
\hline $\mathrm{BMI}, \mathrm{kg} / \mathrm{m}^{2}$, median [IQR] & 9611 & $29.0[25.8-33.1]$ & 3514 & $28.9[25.7-33.1]$ & 6097 & $29.1[25.8-33.1]$ \\
\hline Systolic blood pressure, mmHg, median [IQR] & 9618 & $130.0[120.0-140.0]$ & 3531 & 130.0 [120.0-142.0] & 6087 & $130.0[120.0-140.0]$ \\
\hline Diastolic blood pressure, mmHg, median [IQR] & 9616 & $78.0[70.0-82.0]$ & 3529 & $76.0[70.0-81.0]$ & 6087 & $80.0[70.0-83.0]$ \\
\hline Total cholesterol, mmol/L, median [IQR] & 8272 & $4.34[3.68-5.14]$ & 3001 & $4.05[3.39-4.82]$ & 5271 & $4.51[3.83-5.26]$ \\
\hline LDL cholesterol, mmol/L, median [IQR] & 8090 & $2.39[1.81-3.08]$ & 2924 & $2.12[1.62-2.77]$ & 5166 & $2.54[1.98-3.19]$ \\
\hline HDL cholesterol, mmol/L, median [IQR] & 7965 & $1.15[0.98-1.40]$ & 2907 & $1.11[0.93-1.32]$ & 5058 & $1.18[0.99-1.42]$ \\
\hline Triglyceride, mmol/L, median [IQR] & 8466 & $1.60[1.13-2.27]$ & 3082 & $1.61[1.14-2.30]$ & 5384 & $1.59[1.13-2.25]$ \\
\hline eGFR, mL/min/1.73 m², n (\%) & 7923 & & 2888 & & 5035 & \\
\hline$>89$ & & $2746(34.7)$ & & $707(24.5)$ & & $2039(40.5)$ \\
\hline$>59-89$ & & $3512(44.3)$ & & $1293(44.8)$ & & $2219(44.1)$ \\
\hline$>29-59$ & & $1450(18.3)$ & & $757(26.2)$ & & $693(13.8)$ \\
\hline$\leq 29$ & & $215(2.7)$ & & $131(4.5)$ & & $84(1.7)$ \\
\hline Albuminuriaa, $\mathrm{n}(\%)$ & 6482 & & 2433 & & 4049 & \\
\hline Normal-mildly increased & & $4338(66.9)$ & & $1396(57.4)$ & & $2942(72.7)$ \\
\hline Microalbuminuria & & $1607(24.8)$ & & $774(31.8)$ & & $833(20.6)$ \\
\hline Macroalbuminuria & & $537(8.3)$ & & $263(10.8)$ & & $274(6.8)$ \\
\hline Medical history of hypertension, yes, n (\%) & 9643 & $6756(70.1)$ & 3522 & $2918(82.9)$ & 6121 & $3838(62.7)$ \\
\hline Familial hypercholesterolemia, yes, n (\%) & 6634 & $676(10.2)$ & 2342 & $246(10.5)$ & 4292 & $430(10.0)$ \\
\hline Retinopathy, n (\%) & 9818 & & 3578 & & 6240 & \\
\hline Yes & & $1455(14.8)$ & & $725(20.3)$ & & $730(11.7)$ \\
\hline Yes (referred by participant) & & $399(4.1)$ & & $144(4.0)$ & & $255(4.1)$ \\
\hline No & & $7964(81.1)$ & & $2709(75.7)$ & & $5255(84.2)$ \\
\hline Nephropathy, n (\%) & 9818 & & 3579 & & 6239 & \\
\hline Yes & & $1771(18.0)$ & & $917(25.6)$ & & $854(13.7)$ \\
\hline Yes (referred by participant) & & $337(3.4)$ & & $128(3.6)$ & & $209(3.3)$ \\
\hline No & & $7710(78.5)$ & & $2534(70.8)$ & & $5176(83.0)$ \\
\hline Neuropathy, n (\%) & 9817 & & 3577 & & 6240 & \\
\hline Yes & & $1774(18.1)$ & & $867(24.2)$ & & $907(14.5)$ \\
\hline Yes (referred by participant) & & $459(4.7)$ & & $168(4.7)$ & & $291(4.7)$ \\
\hline No & & $7584(77.3)$ & & $2542(71.1)$ & & $5042(80.8)$ \\
\hline Smoking status, n (\%) & 9725 & & 3547 & & 6178 & \\
\hline Current & & $1322(13.6)$ & & $465(13.1)$ & & 857 (13.9) \\
\hline Previous & & $2613(26.9)$ & & $1268(35.7)$ & & $1345(21.8)$ \\
\hline Never & & $5790(59.5)$ & & $1814(51.1)$ & & $3976(64.4)$ \\
\hline
\end{tabular}


Table 1 (continued)

\begin{tabular}{|c|c|c|c|c|c|c|}
\hline \multirow[t]{3}{*}{ Characteristic } & \multirow{2}{*}{\multicolumn{2}{|c|}{$\begin{array}{l}\text { Overall } \\
\mathrm{N}=9823\end{array}$}} & \multicolumn{4}{|c|}{ By CVD status } \\
\hline & & & \multicolumn{2}{|c|}{$\begin{array}{l}\text { CVD } \\
\mathrm{n}=3582\end{array}$} & \multicolumn{2}{|c|}{$\begin{array}{l}\text { Non-CVD } \\
n=6241\end{array}$} \\
\hline & $\mathbf{n}$ & Data & $\mathbf{n}$ & Data & $\mathbf{n}$ & Data \\
\hline Duration of smoking ${ }^{b}$, years, median [IQR] & 3733 & $28.0[15.0-39.0]$ & 1646 & $30.0[20.0-40.0]$ & 2087 & $25.0[15.0-35.0]$ \\
\hline Physical activityc', days per week, n (\%) & 7492 & & 2763 & & 4729 & \\
\hline $0-1$ & & $3599(48.0)$ & & $1515(54.8)$ & & $2084(44.1)$ \\
\hline $2-3$ & & $1613(21.5)$ & & $497(18.0)$ & & $1116(23.6)$ \\
\hline $4-5$ & & $883(11.8)$ & & $264(9.6)$ & & $619(13.1)$ \\
\hline $6-7$ & & $1397(18.6)$ & & $487(17.6)$ & & $910(19.2)$ \\
\hline
\end{tabular}

To convert the values for glucose to $\mathrm{mg} / \mathrm{dL}$, divide by 0.0555 . To convert the values for cholesterol to $\mathrm{mg} / \mathrm{dL}$, divide by 0.0259 . To convert the values for triglycerides to $\mathrm{mg} / \mathrm{dL}$, divide by 0.0113 . Data that were missing from the medical record but verbally confirmed by a participant were coded as 'referred by participant'. Data were not weighted

${ }^{a}$ Defined as: normal-mildly increased, urinary excretion $<30 \mathrm{mg} / 24 \mathrm{~h}$ or UACR $<30 \mathrm{mg} / \mathrm{g}$; microalbuminuria, urinary excretion $30-299 \mathrm{mg} / 24 \mathrm{~h}$ or UACR $30-299 \mathrm{mg} / \mathrm{g}$; macroalbuminuria, urinary excretion $\geq 300 \mathrm{mg} / 24 \mathrm{~h}$ or UACR $\geq 300 \mathrm{mg} / \mathrm{g}$

${ }^{b}$ Only applies to participants categorized as current or previous smokers

${ }^{\mathrm{C}}$ Days with $\geq 30 \mathrm{~min}$ of moderate activity

$B M /$ body mass index, CVD cardiovascular disease, eGFR estimated glomerular filtration rate, $F P G$ fasting plasma glucose, $H b A_{1 c}$ glycated hemoglobin, $H D L$ highdensity lipoprotein, IQR interquartile range, $L D L$ low-density lipoprotein, UACR urinary albumin to creatinine ratio

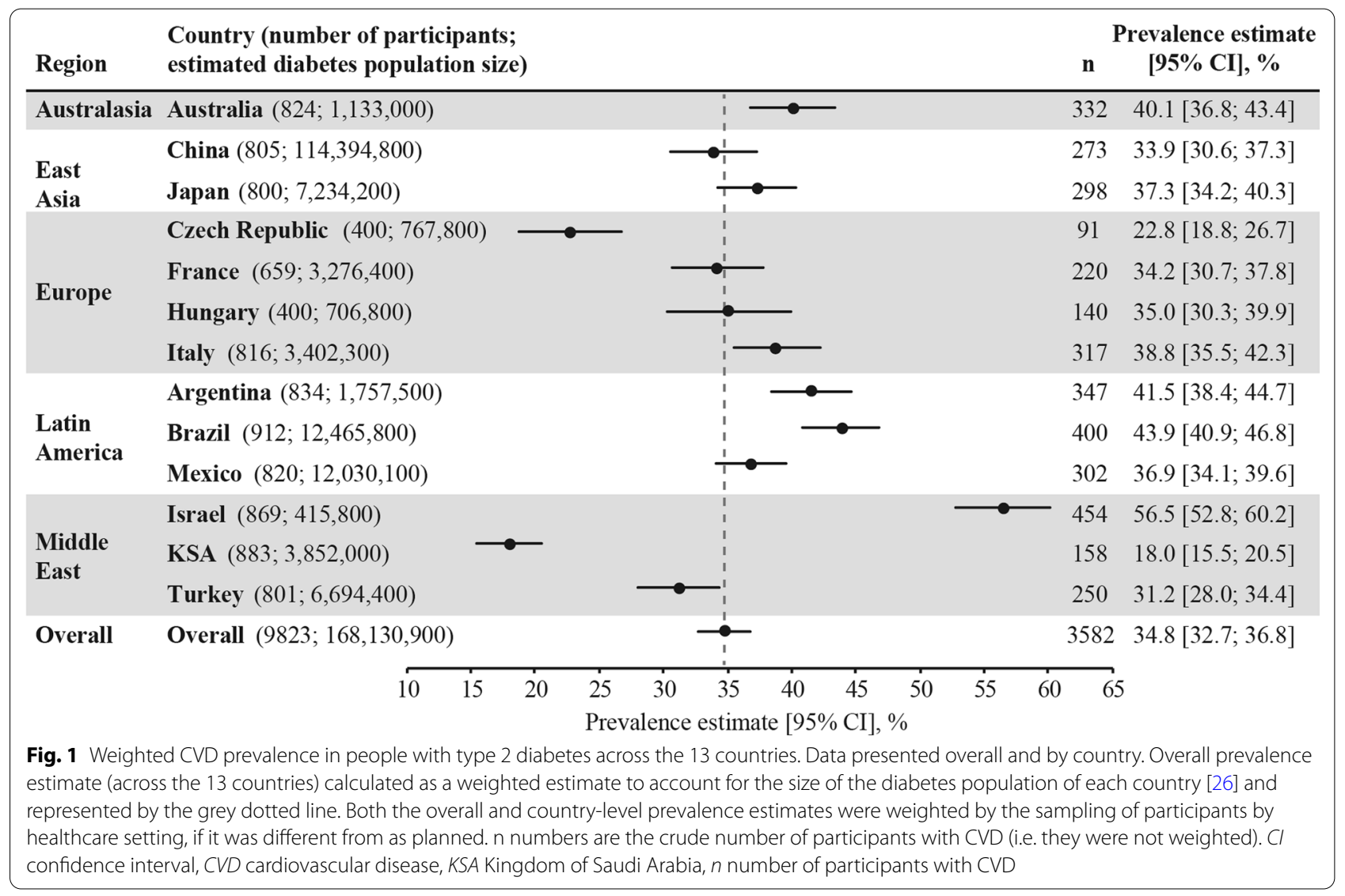




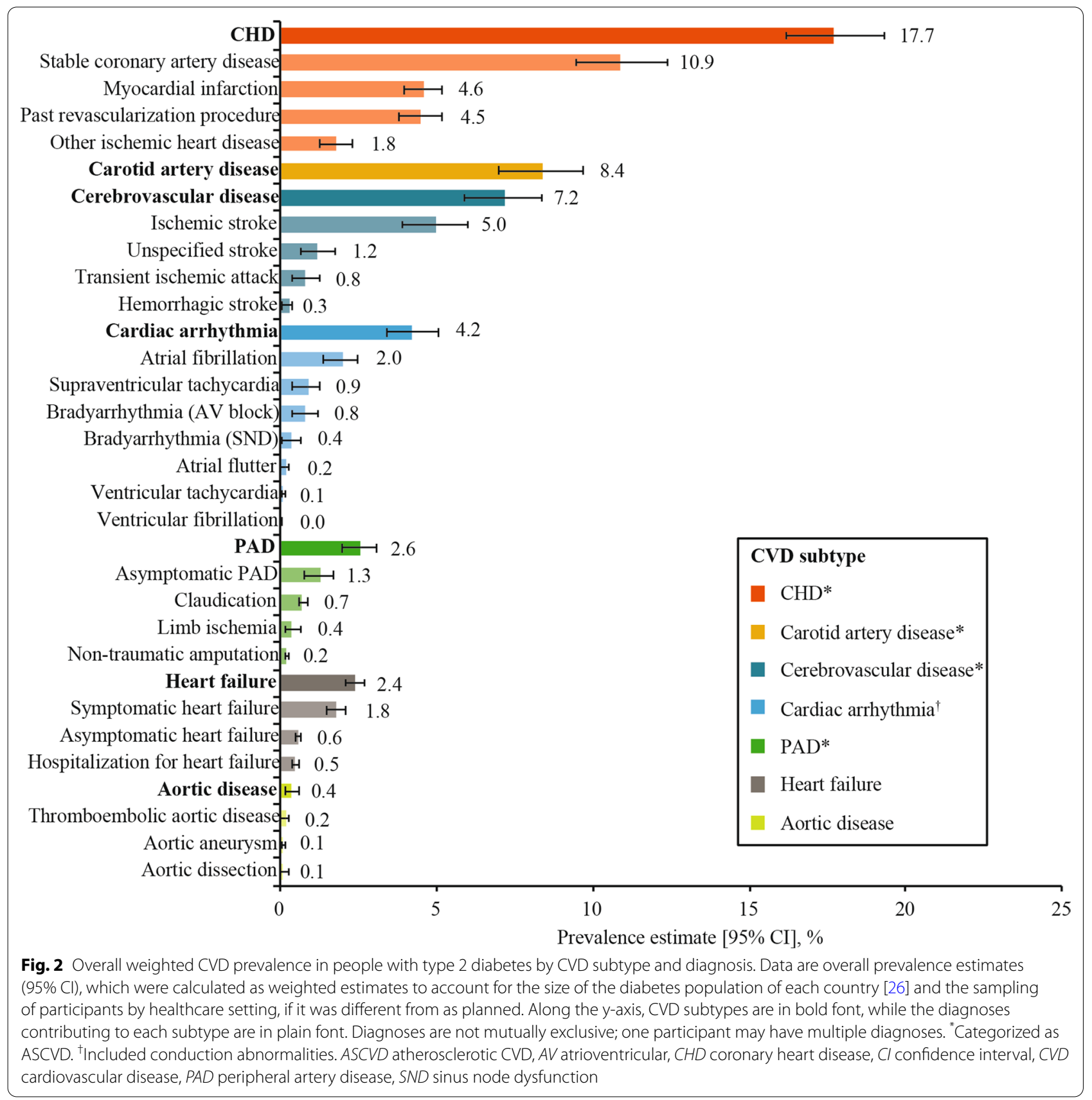

vs 9.8 years) (Table 1 ; not statistically analyzed). In comparison with the non-CVD group, a higher proportion of participants in the CVD group had diagnosed hypertension ( $82.9 \%$ vs $62.7 \%$ ), renal dysfunction (microalbuminuria, $31.8 \%$ vs $20.6 \%$; macroalbuminuria, $10.8 \%$ vs $6.8 \%$; eGFR $\leq 59 \mathrm{~mL} / \mathrm{min} / 1.73 \mathrm{~m}^{2}, 30.7 \%$ vs $15.4 \%$ ), were current or previous smokers (48.9\% vs $35.6 \%)$, and reported low physical activity (0-1 day of physical activity per week, $54.8 \%$ vs $44.1 \%$ ) (Table 1 ; additional data in
Additional file 1: Table S3). In contrast, median serum low-density lipoprotein cholesterol concentrations were lower in the CVD group versus the non-CVD group $(2.12 \mathrm{mmol} / \mathrm{L}$ vs $2.54 \mathrm{mmol} / \mathrm{L})$. The proportion of participants with microvascular complications was higher in the CVD group compared with the non-CVD group (retinopathy: $24.3 \%$ vs $15.8 \%$; nephropathy: $29.2 \%$ vs 17.0\%; neuropathy: $29.0 \%$ vs $19.2 \%$ ). 

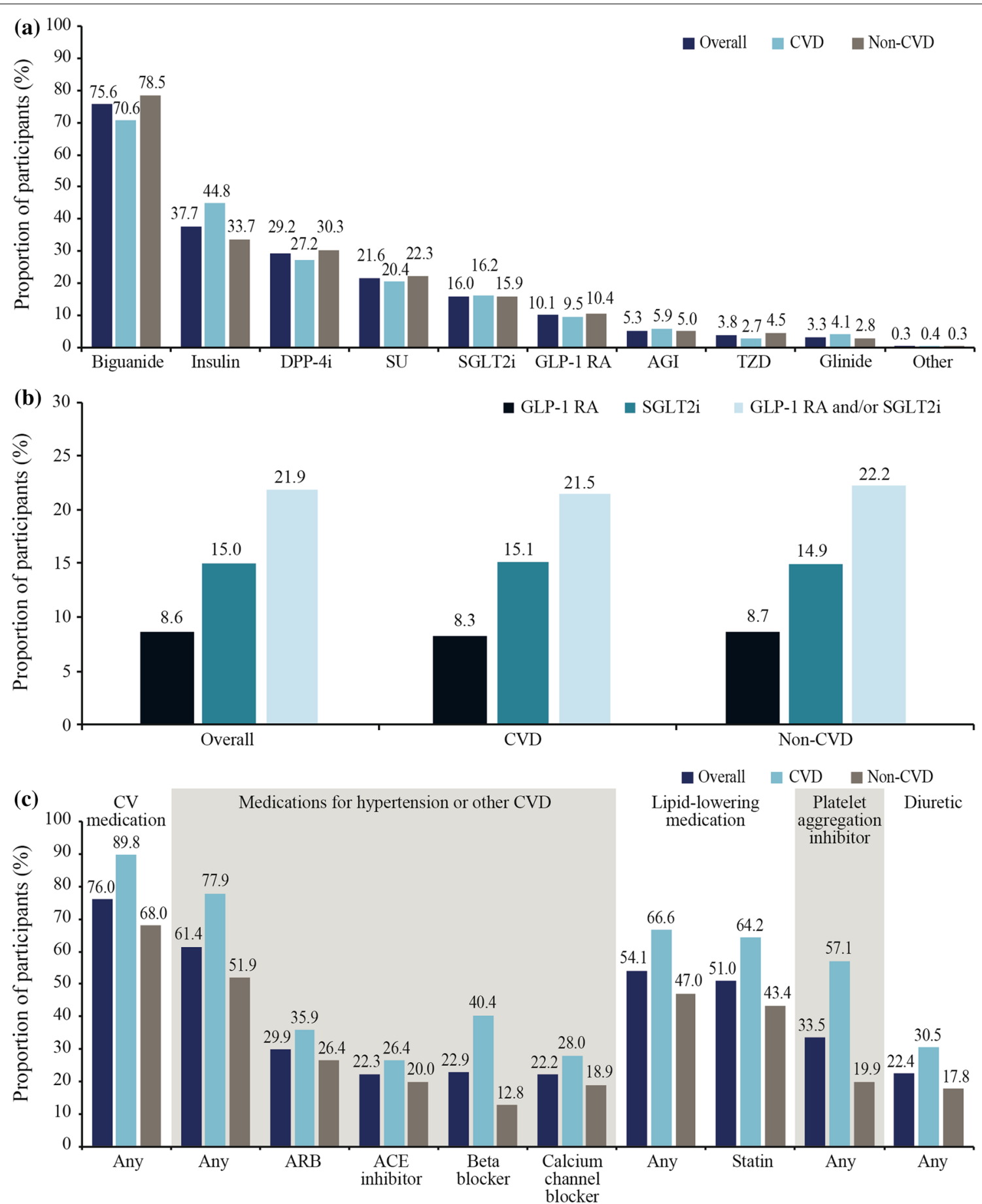

Fig. 3 Use of a GLAs, $\mathbf{b}$ GLAs with demonstrated CV benefit and $\mathbf{c}$ selected CV medications. Data are \% and were not weighted. Data are presented for the CAPTURE study sample overall and stratified by CVD status. In b. GLAs with demonstrated CV benefit were defined per 2020 American Diabetes Association guidelines [19] as GLP-1 RAs: dulaglutide, liraglutide, and semaglutide; and SGLT2is: canagliflozin, dapagliflozin, and empagliflozin. ACE angiotensin-converting enzyme, $A G l$ alpha glucosidase inhibitor, $A R B$ angiotensin II receptor blocker, CV cardiovascular, CVD cardiovascular disease, DPP-4i dipeptidyl peptidase-4 inhibitor, GLA blood glucose-lowering agent, GLP-1 RA glucagon-like peptide-1 receptor agonist, SGLT2i sodium-glucose co-transporter-2 inhibitor, SU sulfonylurea, TZD thiazolidinedione 


\section{GLAs by CVD status}

In total, $96.6 \%$ of the overall study sample were receiving at least one GLA from any class. Biguanides were less frequently prescribed in the CVD group than the non-CVD group (70.6\% vs $78.5 \%)$, whereas insulin use was more common in the CVD group than in the nonCVD group (44.8\% vs $33.7 \%$ ) (Fig. 3a). In total, $21.9 \%$ of participants were prescribed a GLA with demonstrated $\mathrm{CV}$ benefit, and this was similar in the CVD and nonCVD groups (21.5\% vs 22.2\%) (Fig. 3b). SGLT2is were more frequently used than GLP-1 RAs (15.0\% vs $8.6 \%)$ in the overall study sample, with a similar use of both therapeutic classes across the CVD and non-CVD groups.

In exploratory analyses, 2051 and 2498 participants from the non-CVD group were classified as having high CVD risk using some of the REWIND and DECLARETIMI 58 criteria, respectively. In total, $22.2 \%(n=456)$ of participants with high CVD risk according to REWIND criteria and $21.6 \%(n=540)$ of participants with high CVD risk according to DECLARE-TIMI 58 criteria were using a GLA with demonstrated CV benefit.

\section{CV medications by CVD status}

Overall, 7461 participants (76.0\%) were receiving any CV medication and it was more common in the CVD group than in the non-CVD group ( $89.8 \%$ vs $68.0 \%)$, with the same pattern across CV medication classes (Fig. 3c; not statistically analyzed). In the CVD group, statins were the most frequently utilized CV medications (64.2\%), followed by beta-blockers (40.4\%), acetylsalicylic acid (39.0\%), angiotensin II receptor blockers (ARBs; 35.9\%), calcium channel blockers (28.0\%), and angiotensinconverting enzyme (ACE) inhibitors (26.4\%). In the non-CVD group, statin use also predominated (43.4\%), followed by ARBs (26.4\%), ACE inhibitors (20.0\%), acetylsalicylic acid (19.1\%), calcium channel blockers (18.9\%), and beta-blockers (12.8\%). Among participants at high CVD risk in the non-CVD group, statins were most frequently used (43.4\%), followed by ARBs (30.3\%), calcium channel blockers (24.3\%), ACE inhibitors (23.6\%), acetylsalicylic acid (23.3\%), and beta-blockers $(14.0 \%)$.

\section{Vascular areas affected according to gender}

When analyzing the number of affected vascular areas in participants with CVD, data were available for 1134 women and 1940 men. The proportion of participants with one vascular area affected was similar among men and women, although men were more likely to have more vascular areas involved than women (Additional file 1: Fig. S4).
PORs for CVD adjusted for age, gender, and clinical parameters

Differences in age and gender explained some of the variation in CVD prevalence between countries, as PORs in model 1 (age- and gender-adjusted) were closer to 1.00 for some countries (Argentina, Australia, China, Israel, Kingdom of Saudi Arabia, and Turkey) than in the unadjusted model (Additional file 1: Fig. S5). Adjustment for additional clinical parameters (model 2) appeared to explain further variation in CVD prevalence between countries observed in the unadjusted model, with PORs closer to 1.00 for all countries except China, France, and Mexico. However, there were still significant differences in CVD prevalence for some countries in comparison with the overall study sample after further adjustment, specifically lower odds of CVD for Kingdom of Saudi Arabia, Czech Republic, and France, in addition to higher odds of CVD for Israel, Brazil, and China ( $<<0.05$ for all comparisons; Additional file 1: Fig. S5). Findings from these adjusted analyses were consistent with sensitivity analyses without imputation of missing data (Additional file 1: Fig. S6) and including eGFR (both with and without imputation of missing data) (Additional file 1: Fig. S7).

\section{Discussion}

The overall weighted prevalence of CVD ascertained using standardized methodology in the present multinational study of adults with T2D from primary or specialist care settings was estimated at $34.8 \%$. ASCVD accounted for most $(85.8 \%)$ of this disease burden, with stable coronary artery disease, carotid artery disease, and stroke the major components.

The 2019 CAPTURE findings are in accordance with those reported by Einarson and colleagues [16] in their systematic literature review of 57 studies from 25 countries involving over 4.5 million adults with T2D conducted between 1987 and 2015, which estimated a CVD prevalence of 32.2\% [16]. Our weighted prevalence estimate for ASCVD was also similar to that in the systematic review (31.8\% vs $29.1 \%$, respectively) [16]. Other available comparative data are from individual countries not included in CAPTURE. Nevertheless, our CVD prevalence estimate aligns with CVD data linked to hospital admissions for nearly 250,000 Scottish patients with T2D $(32.5 \%)$ [14]. However, it is higher than that reported in a primary care survey of over 17,000 Danish patients with T2D (21.4\%) [11], and our weighted ASCVD prevalence estimate is lower than the $45.2 \%$ reported in a cross-sectional study of over 1.2 million adults with T2D from a US claims database in 2015 [10]. In addition to potential geographical differences, direct comparisons between 
studies are complicated by heterogeneity in factors such as participant selection, study design, timing, and methods of CVD ascertainment. Nevertheless, the CAPTURE data, collected using standardized methodology across 13 countries, make progress in addressing the need for more uniform epidemiological data relating to the global CVD burden [29].

In the present study, post hoc analyses were carried out to identify explanations for the between-country variation in CVD estimates. Differences in age and gender accounted for some of the observed variation between countries, while adjustment for additional clinical parameters further attenuated individual country differences from the pooled estimate. However, even after additional adjustment for potentially confounding parameters, there were significantly lower odds of CVD in the Kingdom of Saudi Arabia, Czech Republic, and France, contrasting with significantly higher odds in Israel, Brazil, and China, as compared with the overall CAPTURE study sample. These between-country differences might reflect variation in the sites selected, genetic and/or lifestyle factors, healthcare accessibility and delivery, CV medication use, medical record characteristics, CVD screening practices, and even competing risk of death from non-diabetes-related causes [30, 31], factors that were not directly addressed in CAPTURE. Our country-level CVD prevalence estimates are similar to those reported by Einarson and colleagues [16] in their systematic literature review, wherein the prevalence in China was $33.9 \%$ in CAPTURE vs $28.4 \%$ in the systematic literature review. However, there was variation among the CVD estimates for the other countries in CAPTURE with outlying odds when compared with available data (Saudi Arabia: 18.0\% vs $30.0 \%$; France: $34.2 \%$ vs $53.9 \%$; Brazil: $43.9 \%$ vs $27.5 \%$, all for CAPTURE vs the systematic literature review [16], respectively). A detailed exploration of outliers was beyond the scope of the present study. With a large diabetes population, China accounted for most of the weighting and may have substantially influenced the results. However, post hoc analyses excluding China found that the overall weighted CVD prevalence was only marginally higher (1.8 percentage points) for the remaining 12 countries, despite a more pronounced influence of China on the weighted heart failure prevalence, which was 4.2 percentage points higher for the remaining 12 countries. This large variation in heart failure prevalence may be attributed to underreporting and missed diagnoses, which may vary considerably between countries, depending on their screening and diagnostic capabilities [32].

Motivated by evidence that ASCVD is largely preventable, many countries are starting to implement policies and practices that aim to decrease this burden in people at high risk, including those with T2D [29, 33]. Effective action to reduce the global burden of CVD in people with T2D requires reliable data on prevalence, risk factors, medication use, and the barriers to prevention and treatment [34]. Our findings indicate that fewer than one in four adults with T2D and established CVD use a GLA with demonstrated CV benefit. In 2015, when relatively few relevant $\mathrm{CV}$ outcome trial data were available, an understandably lower use of GLP-1 RAs (7.9\%) and SGLT2is (8.8\%) was reported in over 500,000 US adults with T2D and established ASCVD [10].

Additionally, our findings indicate that a relatively low proportion of adults with T2D without established CVD but at high CVD risk in CAPTURE - approximately 20\% - used a GLA with demonstrated CV benefit. This has potential clinical implications as REWIND found a nonsignificant trend to fewer major adverse CV events with dulaglutide versus placebo in patients with $\mathrm{CV}$ risk factors but without CVD (hazard ratio [HR] 0.87, 95\% CI 0.74-1.02) [27], and DECLARE-TIMI 58 reported a nonsignificant trend towards a lower rate of a composite of $\mathrm{CV}$ death or hospitalization for heart failure with dapagliflozin versus placebo in the same subgroup (HR 0.84, 95\% CI 0.67-1.04) [28].

The proportions of patients using GLAs with demonstrated CV benefit may change with the implementation and influence of recent updates to diabetes/cardiology guidelines that now recommend a GLP-1 RA or SGLT2i with demonstrated CV benefit as first- or second-line GLA in people with T2D and established CVD or at high/very high CVD risk [5-7]. The future impact of these updates on real-world clinical practice will be of interest, and our contemporary data provide a benchmark against which relevant trends can be monitored. Indeed, regional differences in diabetes and cardiology treatment guidelines, as well as approvals and reimbursement of individual medications including GLAs, are also likely to influence the use of GLAs with CV benefit at a country level. For example, in Brazil, physicians have the ability to prescribe any class of GLA and are limited only by financial considerations. In France, SGLT2is where not approved for use at the time of the CAPTURE study, and even today, GPs have the ability to prescribe GLP-1 RAs but are only permitted to renew prescriptions of SGLT2is after they have been initiated by a specialist. In the Kingdom of Saudi Arabia, the availability of GLP-1 RAs and SGLT2i GLAs is dependent on the purchasing decisions, policies and prescribing privileges in individual governmental hospitals.

A treatment gap exists for other evidence-based therapies. For example, a smaller than recommended proportion of participants with established CVD in CAPTURE were using statins $(64.2 \%)$ or acetylsalicylic acid (39.0\%) $[6,35]$. In patients with known CVD, acetylsalicylic acid 
reduces $\mathrm{CV}$ events, with the benefits outweighing the risk of major bleeding [36]. Statins have also been found to be effective in reducing $\mathrm{CV}$ events in patients with or without established CVD [37]. The CAPTURE data highlight the potential for improved use of non-glycemic $\mathrm{CV}$ pharmacotherapy for people with T2D and CV risk factors. Notably, glycemia, as assessed by $\mathrm{HbA}_{1 \mathrm{c}}$, was relatively well controlled in the overall CAPTURE study sample (median $7.3 \%$ [ $56 \mathrm{mmol} / \mathrm{mol}]$ ). This is in alignment with the $\mathrm{HbA}_{1 \mathrm{c}}$ levels reported by similar studies that estimated CVD prevalence in patients with T2D in primary care (mean $6.9 \%$ [52 $\mathrm{mmol} / \mathrm{mol}])$ [11] and admitted to hospital (median 7.2\% [55 mmol/mol]) [14], albeit in other European settings.

The CAPTURE study has several strengths. It was cross-sectional and multinational in design, with consistent methodology for data collection across different healthcare systems through use of a standardized electronic case report form. Participants were recruited from both primary and specialist care. Broad inclusion criteria were used to ensure that the study sample was as representative as possible of the general adult T2D population, with implications for the generalizability of the findings. As evidenced by key demographic and clinical characteristics, the participants spanned a wide spectrum of disease. Our findings provide contemporary prevalence estimates for several non-US countries where up-todate data were limited or absent [16]. Furthermore, our findings may provide valuable background data for local healthcare payers and policy makers to assist with evaluating strategies to reduce CVD risk in adults with T2D. The application of our findings to the design of CV outcome trials in the study countries could enhance the generalizability of their results by informing trial entry criteria and aiming to increase the representative nature of the trial sample compared with the general T2D population.

The present study has limitations. It is possible that our prevalence data are overestimates, as there may be a tendency for people with complications to consult their healthcare provider more frequently than the general T2D population. This form of ascertainment bias could explain why the CVD prevalence was similar in primary and specialist care. Additionally, very ill people with relatively high rates of complications including CVD may have been unable to attend routine healthcare visits. We cannot exclude the possibility of consent bias, a potential limitation of any study requiring participants' consent for inclusion, in that individuals willing to participate in the study may not have been fully representative of the general population of patients with T2D. Medication use may have been influenced by country-specific guidelines for management of patients with T2D and between-country differences in approvals and reimbursement of individual medications and GLA classes. Furthermore, our study was non-interventional and did not mandate screening for, or adjudication of, the presence of complications. Our findings relied on the clinical capabilities and documentation specific to each participant's healthcare setting. The non-interventional nature of CAPTURE meant that some participants may have had undiagnosed or misdiagnosed CVD, particularly in clinics or countries where relevant investigations were not recommended, there was a lack of diagnostic capability, or where medical records were fragmented or incomplete. As it was important to identify a study population representative of patients with T2D regularly attending primary and/or secondary diabetes centers across the different countries and guarantee accuracy in data collection, the choice of participating sites in primary and specialist care was based on recommendations from personnel with knowledge of the local health system who were employed by the sponsor rather than real-world country-specific data, with the possibility of selection bias. However, final participating sites were selected by the contract research organization (and approved by personnel employed by the sponsor) to optimize the accuracy of CVD data collection.

\section{Conclusions}

CAPTURE found that approximately one in three adults with T2D had established CVD. ASCVD accounted for most of this burden, with stable coronary artery disease, carotid artery disease, and stroke being the major contributors. Most participants with CVD were not managed according to the most recent diabetes and cardiology guidelines, implying potential scope for reducing the excess risk through evidence-based interventions. Our data provide a benchmark against which trends can be monitored in order to evaluate the implementation of recent international guidelines.

\begin{abstract}
Abbreviations
ACE: Angiotensin-converting enzyme; ARB: Angiotensin II receptor blocker; ASCVD: Atherosclerotic cardiovascular disease; BMI: Body mass index; CHD: Coronary heart disease; Cl: Confidence interval; CV: Cardiovascular; CVD: Cardiovascular disease; eGFR: Estimated glomerular filtration rate; GLA: Blood glucose-lowering agent; GLP-1 RA: Glucagon-like peptide-1 receptor agonist; $\mathrm{HbA}_{1 \mathrm{c}}$ : Glycated hemoglobin; HR: Hazard ratio; IQR: Interquartile range; PAD: Peripheral artery disease; POR: Prevalence odds ratio; SGLT2i: Sodium-glucose co-transporter-2 inhibitor.
\end{abstract}

\section{Supplementary Information}

The online version contains supplementary material available at https://doi. org/10.1186/s12933-021-01344-0.

Additional file 1. Additional methods, data and analyses and full list of CAPTURE study investigators/collaborators. 


\section{Acknowledgements}

The authors would like to thank the CAPTURE study participants, investigators, and coordinators. The authors are grateful to Anil Dandu, PhD, Novo Nordisk, for review of and input to the manuscript. The authors acknowledge the contributions of Usha K Thamattoor, MA, and Gaurav Chaudhary, MSc, Novo Nordisk, in addition to Ilan Yanuv, MSc, and Aliza Rozenberg, MA, Hadassah Hebrew University Hospital, to the statistical analyses. Medical writing support was provided by Anna Campbell, PhD, and editorial assistance provided by Izabel James, MBBS, both of Ashfield MedComms, an Ashfield Health company, funded by Novo Nordisk.

\section{Previous presentation}

Parts of this study have been presented at the 56th Annual meeting of the European Association for the Study of Diabetes (EASD), Vienna, Austria, 21-25 September 2020.

\section{Authors' contributions}

All authors confirm that they meet the International Committee of Medical Journal Editors (ICJME) uniform requirements for authorship and that they have contributed to critical analysis and interpretation of the data, drafting and/or critically reviewing the article, and sharing the final responsibility for the content of the manuscript, as well as the decision to submit it for publication. OM is the guarantor of this work and, as such, had full access to all the data in the study, and takes responsibility for the integrity of the data and the accuracy of the data analysis. Author contributions are as follows: conceptualization: NAR; methodology: NAR, OM; formal analysis: KTE and MSK; investigation: OM, AA, JLAL, FB, PD, TMED, GD, TH, CL, GTR, SS, KU, and SV; data curation: KTE and MSK; writing/original draft preparation: KTE, NAR, MSK, and OM; writing/review and editing: all authors; visualization: KTE and MSK; supervision: NAR, KTE, and MSK. All authors read and approved the final manuscript.

\section{Funding}

The CAPTURE study was funded by Novo Nordisk A/S. The funding source (Novo Nordisk A/S) participated in the study design, site selection (in collaboration with a contract research organization [CRO]), study coordination, data management, data analysis, and study report preparation. The CRO (Parexel in European countries or ICON in others) was responsible for study coordination and data collection. The manuscript was drafted with input from all authors. All authors had full access to all the data in the study and had final responsibility for the decision to submit for publication.

\section{Availability of data and materials}

The datasets used and/or analyzed during the current study are available from the corresponding author on reasonable request.

\section{Declarations}

\section{Ethics approval and consent to participate}

The study protocols were approved by the appropriate independent ethics committees and relevant institutional review boards. The study was conducted in accordance with the Declaration of Helsinki [17], International Society for Pharmacoepidemiology Good Pharmacoepidemiology Practices [18], and local regulations. All participants provided written informed consent prior to participation.

\section{Consent for publication}

Not applicable.

\section{Competing interests}

Ofri Mosenzon reports advisory board membership from Novo Nordisk, Eli Lilly, Sanofi, Merck Sharp \& Dohme, Boehringer Ingelheim, Novartis, AstraZeneca, and BOL Pharma; speaker's bureau honorarium from AstraZeneca, Novo Nordisk, Eli Lilly, Sanofi, Merck Sharp \& Dohme, Boehringer Ingelheim, and Janssen; and research grants from Novo Nordisk and AstraZeneca. Abdullah Alguwaihes reports speaker's bureau honoraria and advisory board membership from AstraZeneca, Boehringer Ingelheim, Eli Lilly, Novo Nordisk, and Sanofi; in addition to research support from AstraZeneca and Novo Nordisk. Jose Luis Arenas Leon reports advisory board membership from Novo Nordisk, AstraZeneca, Merck Sharp \& Dohme, and Novartis; speakers bureau honorarium from Novo Nordisk, AstraZeneca, Merck Sharp \& Dohme, and Merck; and research grants from Novo Nordisk, AstraZeneca, Amgen, Merck Sharp \& Dohme, Novartis, Sanofi, Takeda, Janssen, and Bristol Myers Squibb. Fahri Bayram reports speaker honoraria and travel sponsorship from Bilim Ilaç, Abbott, Novo Nordisk, Sanofi Genzyme, Pfizer, and Eczacıbaşı Illaç; advisory board and consultancy for Novo Nordisk, Sanofi Genzyme, Abbott, Pfizer, and Bilim Illaç; and participation in clinical trials for Novo Nordisk, Lilly, Sanofi, and Abbott. Patrice Darmon reports honoraria, consultancy, advisory role, lectures, or travel grants from AstraZeneca, Boehringer Ingelheim, Eli Lilly, Merck Sharp and Dohme, Novartis, Novo Nordisk, Sanofi, Mundipharma, and Abbott. Timothy M.E. Davis reports speaker's bureau honoraria and advisory board membership from Eli Lilly, Merck Sharp \& Dohme, Novo Nordisk, and Sanofi; and speaker's bureau honorarium from Boehringer Ingelheim and AstraZeneca. Guillermo Dieuzeide reports speaker's bureau honoraria, advisory board membership, and grants from AstraZeneca, Novo Nordisk, Sanofi Aventis, and Roche Diagnosis. Kristen T. Eriksen Margit S. Kaltoft and Nicolai A. Rhee are employees of Novo Nordisk; Margit S. Kaltoft and Nicolai A. Rhee also own stock and/ or shares in Novo Nordisk. Tianpei Hong reports speaker's bureau honoraria and advisory board membership from AstraZeneca, Boehringer Ingelheim, Eli Lilly, Merck Serono, Merck Sharp \& Dohme, Novartis, Novo Nordisk, and Sanofi. Csaba Lengyel reports honoraria and travel grants from AstraZeneca, Berlin Chemie/A Menarini, Boehringer Ingelheim, EGIS, KRKA, Lilly Hungária Kft., MSD Pharma, Novartis, Novo Nordisk, Pfizer, Richter Gedeon, Sandoz, Sanofi Aventis, Servier, TEVA, and Wörwag Pharma. Giuseppina T. Russo reports speaker's bureau honoraria and advisory board membership from AstraZeneca, Boehringer Ingelheim, Eli Lilly, Merck Sharp \& Dohme, Novartis, Novo Nordisk, and Sanofi. Shinichiro Shirabe reports honoraria from Novo Nordisk Pharma Ltd., Sumitomo Dainippon Pharma, and Merck Sharp \& Dohme. Katherine Urbancova has worked on oral presentations, articles, and advisory boards for Novo Nordisk, Sanofi Aventis, Boehringer Ingelheim, Novartis, Eli Lilly, and Merck. Sergio Vencio reports research or educational grants from Novartis, Novo Nordisk, Boehringer Ingelheim, and Biomm.

\section{Author details}

${ }^{1}$ Diabetes Unit, Department of Endocrinology and Metabolism, Hadassah Medical Center, Faculty of Medicine, Hebrew University of Jerusalem, Hadassah Hebrew University Hospital, PO Box 12000, 91120 Jerusalem, Israel. ${ }^{2}$ King Saud University, King Saud University Medical City, Riyadh, Kingdom of Saudi Arabia. ${ }^{3}$ Centro de Atención E Investigación Cardiovascular del Potosí, San Luis Potosí, Mexico. ${ }^{4}$ Department of Endocrinology and Metabolism, Faculty of Medicine, Erciyes University, Kayseri, Turkey. ${ }^{5}$ Aix Marseille University, INSERM, INRA, C2VN, Marseille, France. ${ }^{6}$ Medical School, University of Western Australia, Fremantle Hospital, Fremantle, Australia. ${ }^{7}$ Centro de Atención Integral en Diabetes, Endocrinología Y Metabolismo, Chacabuco, Buenos Aires, Argentina. ${ }^{8}$ Novo Nordisk A/S, Søborg, Denmark. ${ }^{9}$ Department of Endocrinology and Metabolism, Peking University Third Hospital, Beijing, China. ${ }^{10}$ University of Szeged, Szeged, Hungary. ${ }^{11}$ Novo Nordisk Health Care AG, Zurich, Switzerland. ${ }^{12}$ Department of Clinical and Experimental Medicine, Policlinico Universitario, University of Messina, Messina, Italy. ${ }^{13} \mathrm{H}$. E. C Science Clinic, Yokohama, Japan. ${ }^{14}$ Diabetologická Interní Ambulance S.R.O., Ostrava, Czech Republic. ${ }^{15}$ Instituto de Ciencias Farmaceuticas, Goiânia, Goiás, Brazil.

Received: 7 April 2021 Accepted: 12 July 2021

Published online: 27 July 2021

\section{References}

1. Gerstein HC. Diabetes: dysglycaemia as a cause of cardiovascular outcomes. Nat Rev Endocrinol. 2015;11:508-10.

2. International Diabetes Federation: IDF Diabetes Atlas, 9th edn. Brussels, Belgium: International Diabetes Federation; 2019.

3. Sarwar N, Gao P, Seshasai SR, Gobin R, Kaptoge S, Di Angelantonio E, et al. Diabetes mellitus, fasting blood glucose concentration, and risk of vascular disease: a collaborative meta-analysis of 102 prospective studies. Lancet. 2010;375:2215-22.

4. Seshasai S, Kaptoge S, Thompson A, Di Angelantonio E, Gao P, Sarwar N, et al. Diabetes mellitus, fasting glucose, and risk of cause-specific death. N Engl J Med. 2011:364:829-41.

5. Cosentino F, Grant PJ, Aboyans V, Bailey CJ, Ceriello A, Delgado $V$, et al. 2019 ESC guidelines on diabetes, pre-diabetes, and 
cardiovascular diseases developed in collaboration with the EASD. Eur Heart J. 2020;41:255-323.

6. American Diabetes Association. 10. Cardiovascular Disease and Risk Management: Standards of Medical Care in Diabetes-2021. Diabetes Care. 2021:44:S125-50

7. Arnett DK, Blumenthal RS, Albert MA, Buroker AB, Goldberger ZD, Hahn EJ, et al. 2019 ACC/AHA guideline on the primary prevention of cardiovascular disease: executive summary: a report of the American College of Cardiology/American Heart Association Task Force on Clinical Practice Guidelines. Circulation. 2019;140:e563-95.

8. Buse JB, Wexler DJ, Tsapas A, Rossing P, Mingrone G, Mathieu C, et al. Update to: management of hyperglycemia in type 2 diabetes, 2018. A consensus report by the American Diabetes Association (ADA) and the European Association for the Study of Diabetes (EASD). Diabetes Care. 2018;2020(43):487-93.

9. Harding JL, Pavkov ME, Magliano DJ, Shaw JE, Gregg EW. Global trends in diabetes complications: a review of current evidence. Diabetologia. 2019;62:3-16.

10. Weng W, Tian Y, Kong SX, Ganguly R, Hersloev M, Brett J, et al. The prevalence of cardiovascular disease and antidiabetes treatment characteristics among a large type 2 diabetes population in the United States. Endocrinol Diabetes Metab. 2019;2:e00076.

11. Rungby J, Schou M, Warrer P, Ytte L, Andersen GS. Prevalence of cardiovascular disease and evaluation of standard of care in type 2 diabetes: a nationwide study in primary care. Cardiovasc Endocrinol. 2017;6:145-51.

12. Pugliese G, Solini A, Bonora E, Orsi E, Zerbini G, Fondelli C, et al. Distribution of cardiovascular disease and retinopathy in patients with type 2 diabetes according to different classification systems for chronic kidney disease: a cross-sectional analysis of the renal insufficiency and cardiovascular events (RIACE) Italian multicenter study. Cardiovasc Diabetol. 2014:13:59.

13. Gjelsvik B, Tran AT, Berg TJ, Bakke A, Mdala I, Nokleby K, et al. Exploring the relationship between coronary heart disease and type 2 diabetes: a crosssectional study of secondary prevention among diabetes patients. BJGP Open. 2019;3: bjgpopen18X101636.

14. McGurnaghan S, Blackbourn LAK, Mocevic E, Haagen Panton U, McCrimmon RJ, Sattar N, et al. Cardiovascular disease prevalence and risk factor prevalence in type 2 diabetes: a contemporary analysis. Diabet Med. 2019;36:718-25.

15. Franch-Nadal J, Mata-Cases M, Vinagre I, Patitucci F, Hermosilla E, Casellas $A$, et al. Differences in the cardiometabolic control in type 2 diabetes according to gender and the presence of cardiovascular disease: results from the eControl study. Int J Endocrinol. 2014;2014:131709.

16. Einarson TR, Acs A, Ludwig C, Panton UH. Prevalence of cardiovascular disease in type 2 diabetes: a systematic literature review of scientific evidence from across the world in 2007-2017. Cardiovasc Diabetol. 2018;17:83.

17. World Medical Association. Declaration of Helsinki: ethical principles for medical research involving human subjects. JAMA. 2013;310:2191-4.

18. Guidelines for Good Pharmacoepidemiology Practices (GPP) https:// www.pharmacoepi.org/resources/policies/guidelines-08027/.

19. American Diabetes Association. 10. Cardiovascular Disease and Risk Management: Standards of Medical Care in Diabetes-2020. Diabetes Care. 2020;43:S111-34.

20. Trulicity ${ }^{\circledR}$ Prescribing Information https://www.accessdata.fda.gov/drugs atfda_docs/label/2019/125469s023/bl.pdf. Accessed 24 June 2021.
21. Victoza ${ }^{\circledR}$ Prescribing Information https://www.novo-pi.com/victoza.pdf. Accessed 24 June 2021.

22. Invokana ${ }^{\circledR}$ Prescribing Information http://www.janssenlabels.com/packa ge-insert/product-monograph/prescribing-information/INVOKANA-pi. pdf. Accessed 24 June 2021.

23. Farxig ${ }^{\circledR}$ Prescribing Information https://www.accessdata.fda.gov/drugs atfda_docs/label/2014/202293s003lbl.pdf. Accessed 24 June 2021.

24. Jardiance ${ }^{\circledR}$ Prescribing Information https://docs.boehringer-ingelheim. com/Prescribing\%20Information/PIs/Jardiance/jardiance.pdf. Accessed 24 June 2021.

25. Ozempic ${ }^{\circledR}$ Prescribing Information [https://www.novo-pi.com/ozempic. pdf]. Accessed 24 June 2021.

26. International Diabetes Federation: IDF Diabetes Atlas, 8th edn. Brussels, Belgium: International Diabetes Federation; 2017.

27. Gerstein HC, Colhoun HM, Dagenais GR, Diaz R, Lakshmanan M, Pais $P$, et al. Dulaglutide and cardiovascular outcomes in type 2 diabetes (REWIND): a double-blind, randomised placebo-controlled trial. Lancet. 2019:394:121-30

28. Wiviott SD, Raz I, Bonaca MP, Mosenzon O, Kato ET, Cahn A, et al. Dapagliflozin and Cardiovascular Outcomes in Type 2 Diabetes. N Engl J Med. 2019;380:347-57

29. Barquera S, Pedroza-Tobías A, Medina C, Hernández-Barrera L, BibbinsDomingo K, Lozano R, et al. Global overview of the epidemiology of atherosclerotic cardiovascular disease. Arch Med Res. 2015;46:328-38.

30. Schütte S, Acevedo PNM, Flahault A. Health systems around the world - a comparison of existing health system rankings. J Glob Health. 2018:8:010407.

31. World Health Organization (WHO). Global Atlas on Cardiovascular Disease Prevention and Control. Geneva: World Health Organization; 2011.

32. Boonman-de Winter LJM, Rutten FH, Cramer MJM, Landman MJ, Liem AH, Rutten GEHM, Hoes AW. High prevalence of previously unknown heart failure and left ventricular dysfunction in patients with type 2 diabetes. Diabetologia. 2012, 55(8):2154-2162.

33. World Health Organization (WHO). A Prioritized Research Agenda for Prevention and Control of Noncommunicable Diseases. Geneva: World Health Organization; 2011.

34. Joseph P, Leong D, McKee M, Anand SS, Schwalm JD, Teo K, et al. Reducing the global burden of cardiovascular disease, part 1: the epidemiology and risk factors. Circ Res. 2017;121:677-94.

35. Schnell O, Erbach M, Hummel M. Primary and secondary prevention of cardiovascular disease in diabetes with aspirin. Diab Vasc Dis Res. 2012:9:245-55

36. Baigent C, Blackwell L, Collins R, Emberson J, Godwin J, Peto R, et al. Aspirin in the primary and secondary prevention of vascular disease: collaborative meta-analysis of individual participant data from randomised trials. Lancet. 2009:373:1849-60

37. Baigent C, Blackwell L, Emberson J, Holland LE, Reith C, Bhala N, et al. Efficacy and safety of more intensive lowering of LDL cholesterol: a metaanalysis of data from 170,000 participants in 26 randomised trials. Lancet. 2010;376(9753):1670-81.

\section{Publisher's Note}

Springer Nature remains neutral with regard to jurisdictional claims in published maps and institutional affiliations. 